METALLURGY AND FOUNDRY ENGINEERING - Vol. 37, 2011, No. 1

Jerzy Zych*, Tomasz Snopkiewicz**

\title{
DRYING AND HARDENING OF CERAMIC MOULDS APPLIED IN THE INVESTMENT CASTING TECHNOLOGY - INVESTIGATIONS OF THE PROCESS KINETICS
}

\section{INTRODUCTION}

Requirements of an environment protection concerning limitation of volatile substances emission are forcing changes of several technologies applied in foundry practice. In the first place the application of technologies using easily evaporating substances, including alcohols, is limited. Restrictions concern alcohol protective coatings, which are substituted by water coatings. Ethyl silicate, ceramic sands binding agent, is successively substituted by colloidal silica in investment casting technology.

Drying and the following hardening of layers produced with using binders dissolved in water solutions is a much slower process than the one with alcohol as a solvent. In some processes, as e.g. at drying protective coatings on moulds and cores, these drying procedure can be accelerated either by warming the mould (core) or by blowing warmed air. However, such solution is not possible in the case of the investment casting technology, since mould warming could cause deformation of a wax model. In the transitory period, and that is the actual situation concerning this technology, the alternate building of layers: one with the colloidal binder and the next with ethyl silicate is applied in several industrial plants. This procedure accelerates the drying process, but can not be considered the final solution.

\section{RESULTS OF INVESTIGATIONS}

\subsection{Optimal temperature of ceramic moulds drying}

From the point of view of a drying rate of ceramic moulds, for obtaining high yield in the multilayer moulds production, the optimal temperature could be considered the maximal

* Ph.D., D.Sc., ** M.Sc.: Faculty of Foundry Engineering, AGH University of Science and Technology, Kraków, Poland; e-mail: jzych@agh.edu.pl 
one at which such moulds can be produced. This temperature is, in turn, related to the strength of wax model systems. Investigations were performed to determine the temperature influence on a tensile strength of wax mixtures widely used in the investment casting technology. The obtained results are presented in Figure 1.

These examinations indicate and indirectly confirm industrial practice, that the maximum temperature of drying the successive ceramic layers should not exceed $25{ }^{\circ} \mathrm{C}$. On the basis of the obtained strength results, this temperature should be considered the optimal one for the ceramic moulds drying process in the investment casting technology. This temperature is currently applied in industrial practice.

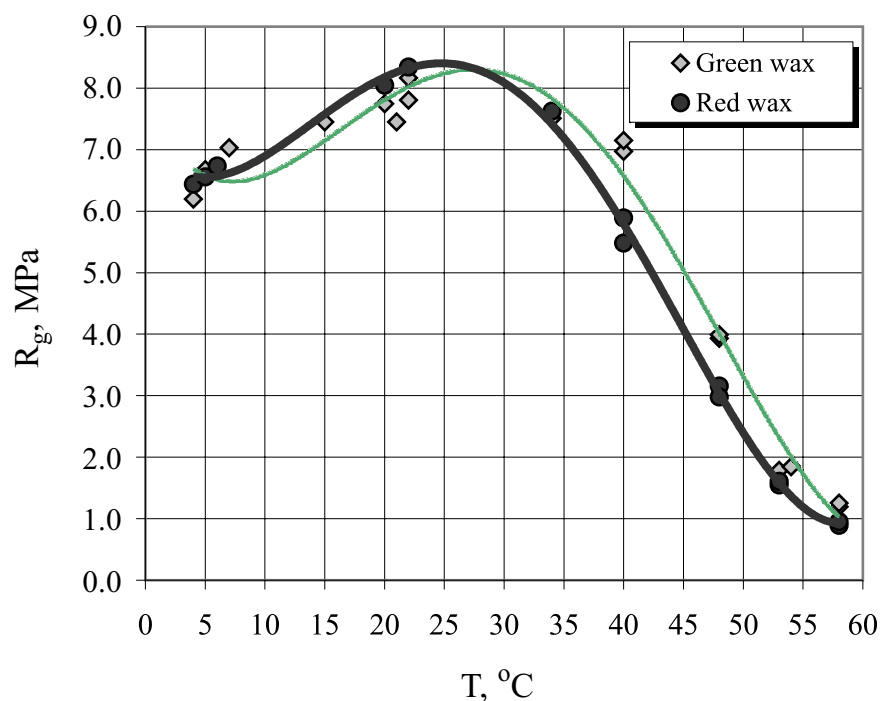

Fig. 1. Influence of a temperature on a tensile strength $R_{g}$ of wax mixtures used in the investment casting technology

\subsection{Investigations of kinetics of ceramic layers drying}

Drying of ceramic layers at a relatively not high temperature $\left(\sim 25^{\circ} \mathrm{C}\right)$ is quite slow. Deposition of the next layer, in the production of a multilayer mould, should be done only when the drying process of the previous one is practically finished (under the applied drying conditions, temperature and air humidity) and the weight loss of the mould being dried is very small. Electrical conductivity measurements or resistance changes of the drying layer were used, up to the present, to determine this moment in the drying procedure. A continuous measurement of the mass (weight) of the drying mould is also a simple method. The ultrasound method was developed recently [1-6]. Within the hereby presented paper measurements were performed by means of all three techniques, including the following simultaneous (parallel) measurements:

- Resistance - weight,

- Resistance - ultrasound. 
Performing parallel (and simultaneous) measurements of the kinetics of the drying process should allow to determine more accurately the time when the single layer is nearly dried as well as should allow to assess the measuring accuracy of individual methods. In addition, it creates the possibility of estimating the suitability of these methods for the technological inspection of the pathway and control of the ceramic moulds drying process.

\subsubsection{Investigations of kinetics by the resistance and weight methods}

The parallel examinations of the ceramic moulds drying process were performed by simultaneous measuring the sample weight (by using balance-dryer) and resistance. The photograph of the experimental stand is shown in Figure 2. The obtained results were recorded on line by means of the computer.
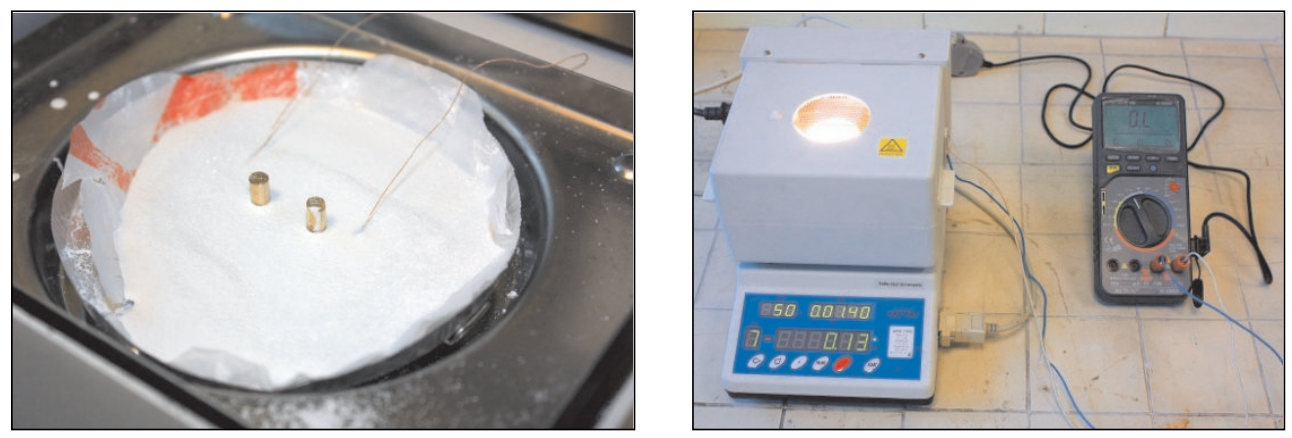

Fig. 2. Probe and the experimental stand for the parallel investigations of the kinetics of ceramic layer drying by the weight and resistance methods

The ceramic moulding sand used in investigations consisted of colloidal silica (Ludox SK or Ludox $\mathrm{AM}$ ) and $\mathrm{Al}_{2} \mathrm{O}_{3}$ flour being a matrix. For sprinkling the ceramic sand layer - deposited on the model - also the $\mathrm{Al}_{2} \mathrm{O}_{3}$ matrix, of an average grain size $d_{L}=0.20 \div 0.30 \mathrm{~mm}$, was used. The aim of these investigations was to determine the course of the sand hardening caused by drying and to find out at what sand moistness an increased resistance occurs.

The idea of measurements carried out by the current technique is based on the determination of the current value in the system, in which the ceramic mould is one of the elements. A constant reference voltage is applied to the drying layer via electrodes and the passage of current values is recorded. The current value is determined by recording the voltage on the resistor connected in series with the drying sand. The current passing through such electric circuit causes the voltage decrease on the measuring resistor, which is recorded. When the layer is drying an amount of electrolyte (water) decreases and to this end the electric resistance in the system increases while the voltage measured on the resistor decreases. Either the voltage or current or resistance of the tested layer can be the measured value. Measuring the resistance was applied in the described examinations of the drying ceramic moulding sand. Out of several methods, the direct measuring of the series resistance was selected on 
account of easiness of its recording. Other methods such as technical or bridge measurements could have been selected but it would render the recording difficult at only insignificant improvements of the measuring accuracy. The performed examinations were of a qualitative character (not quantitative), since at this stage of research they were only aimed at acquainting with the drying effect and relating it to other parameters. The resistance measuring and recording was carried out by means of the multimeter communicating with the computer via the RS232C port. A constant range of measuring resistance of $40 \mathrm{MOhm}$ was established. The results were recorded every 10 seconds.

In case of investigations of the moisture content changes in the drying layer, the resistance creating elements are: the sand layer adjacent to electrodes and the interelectrode layer. The ceramic sand itself, built out of metal oxides, is an insulator - thus, the current is conducted by a solvent - in this case: mineralised water.

All measurements were performed on the insulated base in order to prevent other current passages than only in between electrodes through the ceramic layer. All electrode connections with the measuring system were insulated, only side surfaces of the electrode roll were left active.

Electrodes were made of brass dusted with gold. Such treatment was aimed at minimizing additional electrochemical reactions at the contact: wet sand - electrode. As it can be seen the electrode material did not change visibly the results (comparison between the brass and brass dusted with gold electrodes). According to the second Ohm's law the resistance depends on the electrode surface area. The resistance of the conductor segment (of a constant cross-section) is proportional to the segment length and inversely proportional to the cross-section surface area

$$
R=\rho \frac{l}{S}
$$

where:

$l$ - conductor length,

$S$ - cross-section surface area,

$\rho-$ material conductivity (specific conductance).

The results of the kinetics of drying are presented in Figures 3 and 4 . Since the main aim of investigations was the comparison of weight and resistance change pathways, the measurements were carried out at an increased temperature (for shortening the experiment time). It is seen (Fig. 3), that water losses are, in approximation, linear, and it was only when the drying was in its final phase that the $\mathrm{H}_{2} \mathrm{O}$ content change was much smaller.

Resistance changes of the drying layer are of a different character (Fig. 4). For a long drying period the resistance of the tested layer remains constant and close to zero (for the measuring range of $40 \mathrm{M} \Omega$ ). Barely in the final phase of drying a quite violent increase of the resistance is observed. Probably, the sand layer drying oriented from the outer surface (uncovered) in the direction of the surface adjacent to the model, is the reason of such pathway. A current, regardless of the drying process progressing, is conducted by the near 
model layer up to the moment when in this layer the water content starts also to decrease. Thus it can be concluded, however it still requires further investigations, that the electric method (current) enables to determine - quite well - the end point of the ceramic layer drying.

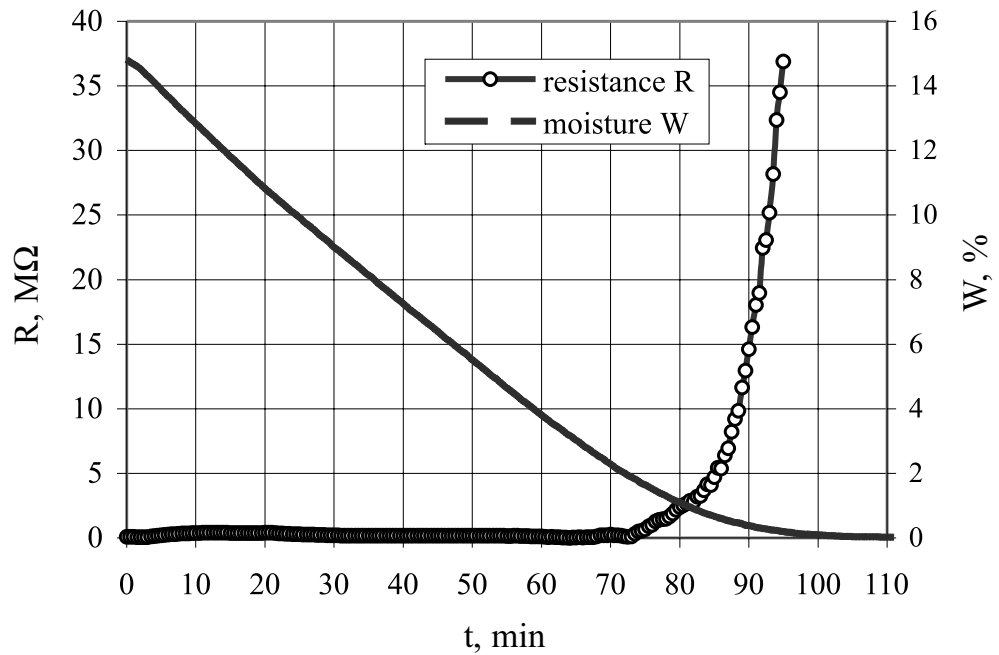

Fig. 3. Pathways of the moisture content and resistance of the ceramic layer

The results presented in Figure 4 allow to notice that the resistance increase occurs when the water content decreases from the initial $16 \%$ to app. $2 \%$. The electric method, which basis on the resistance measurements of the drying ceramic layer, can be used for the determination of the moment when the drying process is nearing its end.

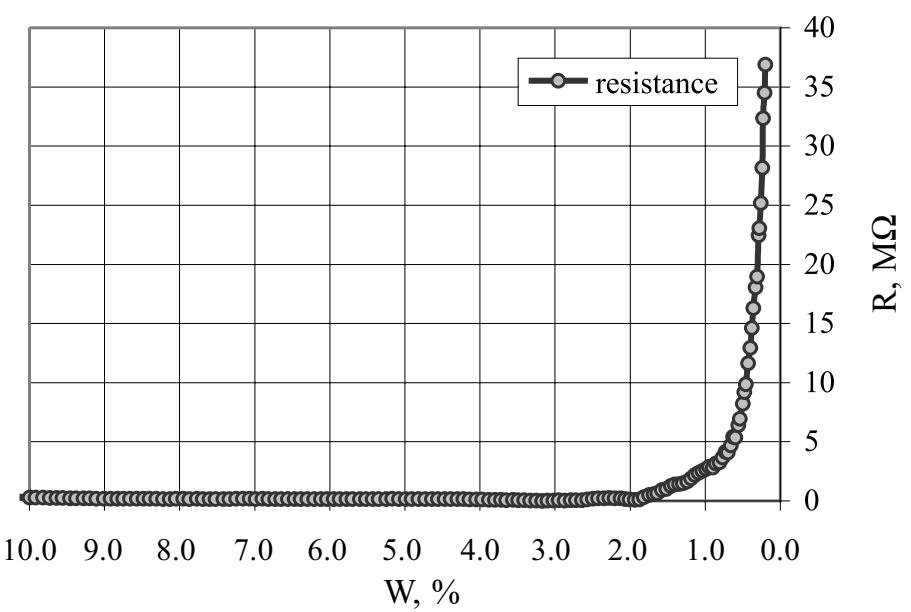

Fig. 4. Dependence between the ceramic sand moisture content and its resistance 


\subsubsection{Investigations of the kinetics of hardening performed by the resistance and ultrasound method}

The essence of investigations by the ultrasound method is the measurement of changes of the dynamic elasticity modulus of the drying ceramic layer. The wet layer, in its initial phase is of a fluid moulding sand consistence. Later on, in the further phase of the drying process, the layer state changes via the viscous-elastic state into the solid one, which is characterised by a significantly higher elasticity (modulus of elasticity $E_{d}$ ). This process causes an increased ultrasound wave propagation velocity in such medium. Equation (2) provides the dependency between the modulus of elasticity and wave velocity

$$
E_{d}=\rho \cdot\left(c_{L}\right)^{2}
$$

where:

$E_{d}-$ dynamic modulus of elasticity,

$\rho$ - density,

$c_{L}-$ wave velocity.

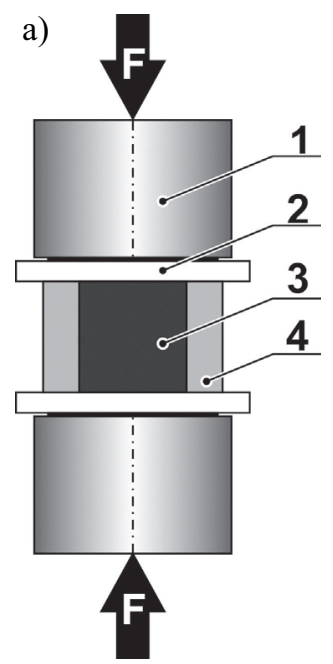

b)

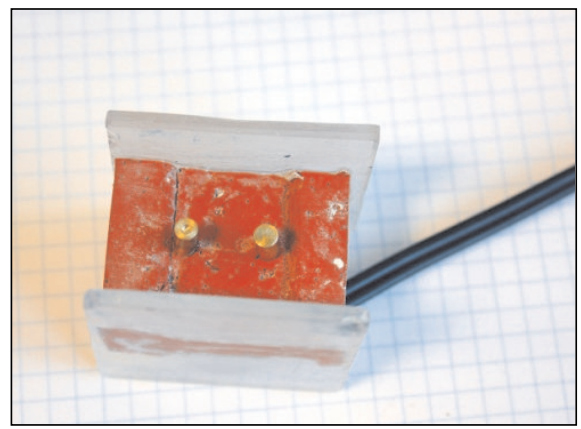

Fig. 5. Ultrasound investigations (a) of the kinetics of the ceramic layers hardening: 1 -ultrasound heads, 2 -plates, 3 -sleeve, 4 -sample of the tested ceramic material and probe prepared (b) for the parallel investigations of the kinetics of the ceramic layers hardening by the ultrasound and electric (resistance) methods

The schematic presentation of the probe for ultrasound testing is given in Figure 5a, while the probe prepared for the parallel ultrasound and resistance investigations is seen in Figure 5b. The multilayer ceramic shell, prepared for ultrasound testing, is deposited on the 
„model” sample - according to the scheme shown in Figure 5a. In order to force the wave propagation through the ceramic layer (being hardened) and to create conditions for measuring the wave velocity in this very layer (and not in the core), the sample core is made of the material which ability for wave suppression is higher, than the material used for shell forming. Admittedly, the wave is propagating via both mediums but the receiving head records the arrival of the first signal. For the wave velocity measuring the time of the wave propagation, from the sending to receiving head, is needed. This time is recorded by the computer in the required time intervals, e.g. every 10 seconds.

The essence of ultrasound investigations allowing to monitor the kinetics of the ceramic layers hardening consists of measurements - in an actual mode - of the medium (being under testing) ability to propagate ultrasound waves. Hardening of the ceramic layer is accompanied by an increase of the propagated wave velocity in the hardening material (medium). The wave velocity increment is a measure of the hardening degree (bonding) increment. Measurements are carried out in the chamber, with the stabilised temperature and air moistness (Fig. 6a and 6b).

a)

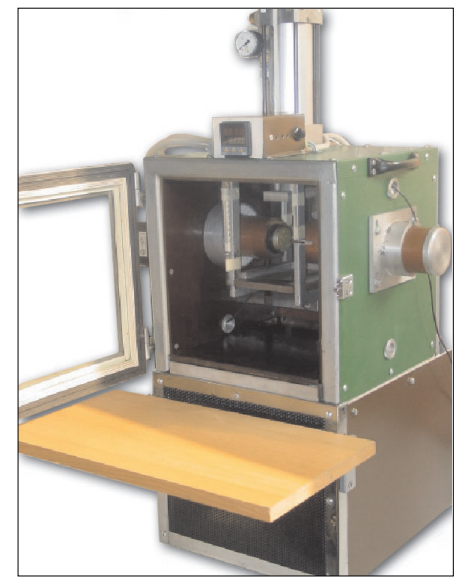

b)

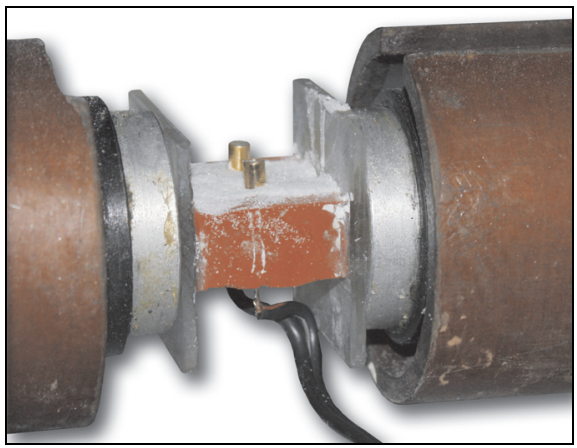

Fig. 6. Chamber of the stabilized temperature (a), applied in ultrasound investigations of the kinetics of hardening of materials with binders and probe with the ceramic moulding sand (b) placed in between ultrasound heads, prepared for investigations of the hardening kinetics

The parallel measurements of the kinetics of the ceramic sand hardening with ultrasound and resistance changes measurements were carried out. The recorded pathways are presented in Figures 7 and 8. The course of the wave velocity changes is of a typical character, indicated in the previous works of the author [3, 4]. In the initial phase, when a water 'excess' evaporates from the ceramic moulding sand, and notwithstanding the sand does not become elastic, the wave velocity does not change. It is seen in Figure 7 that this period under the applied conditions - lasts slightly above 15 minutes. At further drying the visible 
increase of the wave velocity occurs and lasts, in the discussed case, for app. $60 \mathrm{~min}$. Still further drying of the ceramic layer does not cause significant changes neither of the wave velocity nor of the modulus of elasticity.

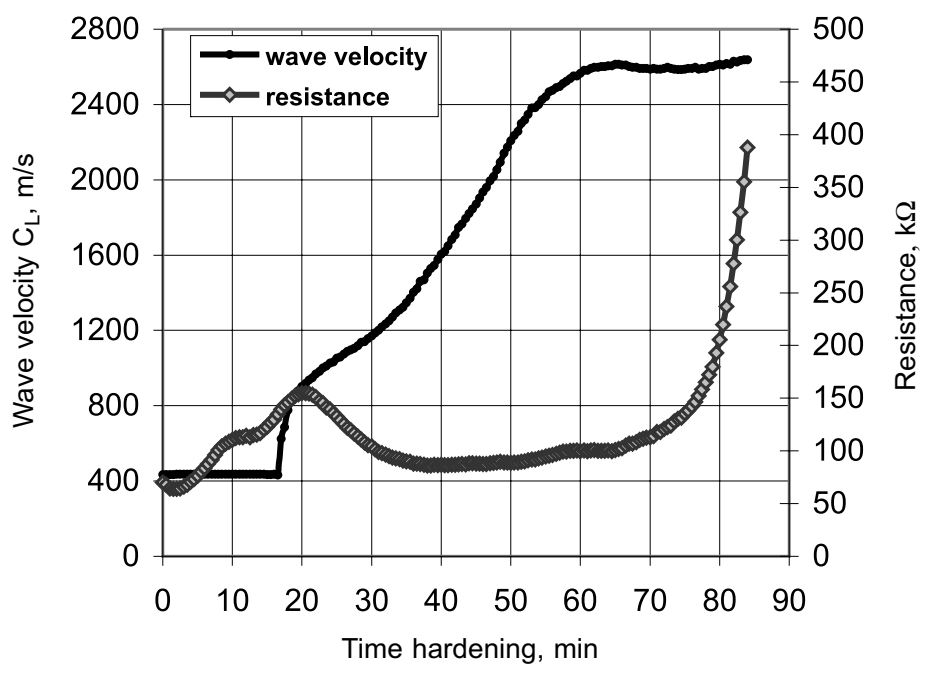

Fig. 7. Influence of the drying time (hardening) of the ceramic moulding sand with colloidal binder (Ludox AM) on the resistance and wave velocity; $\mathrm{Al}_{2} \mathrm{O}_{3}$ was used as a coarse-grained matrix for dusting the fluid moulding sand

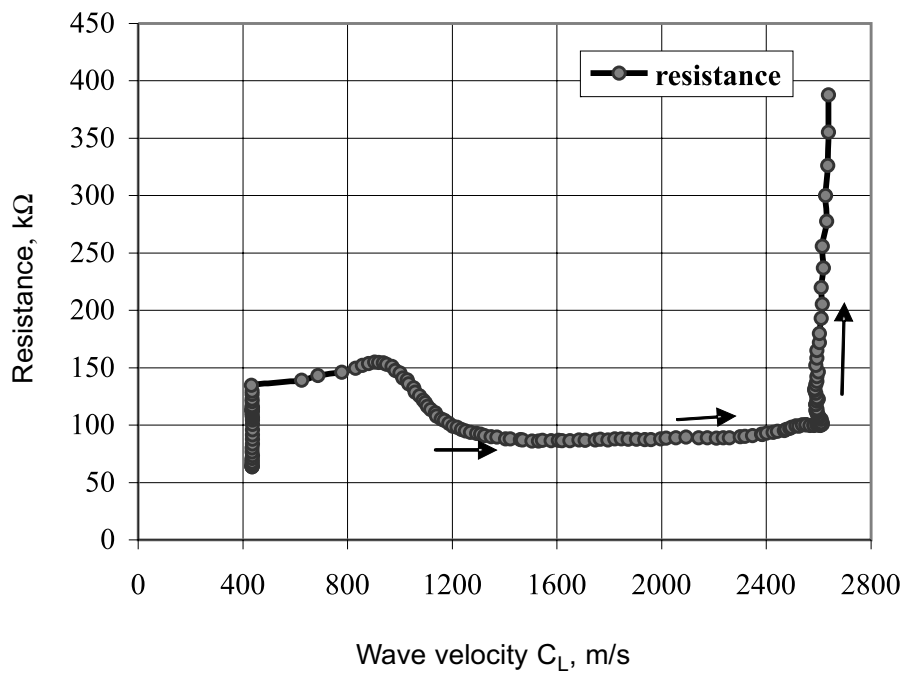

Fig. 8. Dependence between the ultrasound wave velocity and resistance of the ceramic sand with colloidal binder (Ludox AM); $\mathrm{Al}_{2} \mathrm{O}_{3}$ was used as a coarse-grained matrix for dusting the fluid moulding sand. 
Investigations of the ceramic layer resistance lead to slightly different observations. During the first 15 minutes a small increase of the resistance occurs, later on - between 15 and 60 minutes of drying - the resistance is again small and close to the initial one. It was only after app. 60 minutes, when in the ultrasound investigations the wave velocity stabilisation was noticed (sand layer elasticity), a quite fast resistance increment was seen. This period - under the applied condition - lasts slightly above 20 minutes. Juxtaposition of the obtained results of the wave velocity $\left(c_{L}\right)$ and resistance $(R)$ provides the picture shown in Figure 8 . Time is eliminated from graph 7 , since de facto it is situated on the third coordinate of the spatial picture of function: $c_{L}, R=f(\tau)$.

The results juxtapositioned in Figure 8 provide the following pathway of physical property changes of the hardening ceramic moulding sand: in the first phase of drying, when there is an ,excess” of water - the wave velocity is constant $(400 \mathrm{~m} / \mathrm{s})$ and the resistance increases only insignificantly. At further drying (the second period) the layer strengthening starts and is accompanied by the increasing wave velocity. The shell hardening proceeds from the outer (open) surface via the successive, more deeply situated layers. Such is the nature of drying the layers, which are open on one side. During the second period, as it was previously noticed at the weight-resistance investigations, the layer resistance remains at the low and stabilized level. In the final phase, when - as can be assumed - the water loss process spread already across the whole shell thickness, the wave velocity stabilization is observed at the level of maximum values $\left(c_{L} \approx 2.600 \mathrm{~m} / \mathrm{s}\right)$ and the period of the fast resistance increases occurs. The resistance increased to app. $400 \mathrm{kOhm}$ in the final process phase (the resistance value in the performed test depends, among others, on the size and placement of electrodes). This is a period of evaporating of 'left over' water, from the range: $\mathrm{H}_{2} \mathrm{O}<2.0 \%$.

\subsection{Influence of the ceramic mould moisture content $(W)$ on its strength $\boldsymbol{R}_{m}$}

Examinations of changes in the moisture content (W) of the drying layer were supplemented by investigations of its strength as a water content function. In the investment casting technology this interoperation strength is called 'strength in wet condition' or 'green strength'. The obtained results of this strength measurements for the described here ceramic moulding sand are shown in Figure 9. Investigations were made after the deposition of 4 layers. An initial moisture content of such multilayer mould is above $15 \%$. It is seen in Figure 9, that the ceramic mould gains strength when the water content decreases, in practice, below $4.0 \%$, while the visible increases of $R_{m}$ occur only when the water content is below $<2.5-2.0 \%$. Within this range the decisive resistance increase of the ceramic layer is observed as well as maximum velocities of the ultrasound wave are obtained (maximum values of the modulus of elasticity $E_{d}$ ). Within the same wetness range a distinct decrease of the drying rate is observed, which indicates that the physical character of the drying process has changed. 


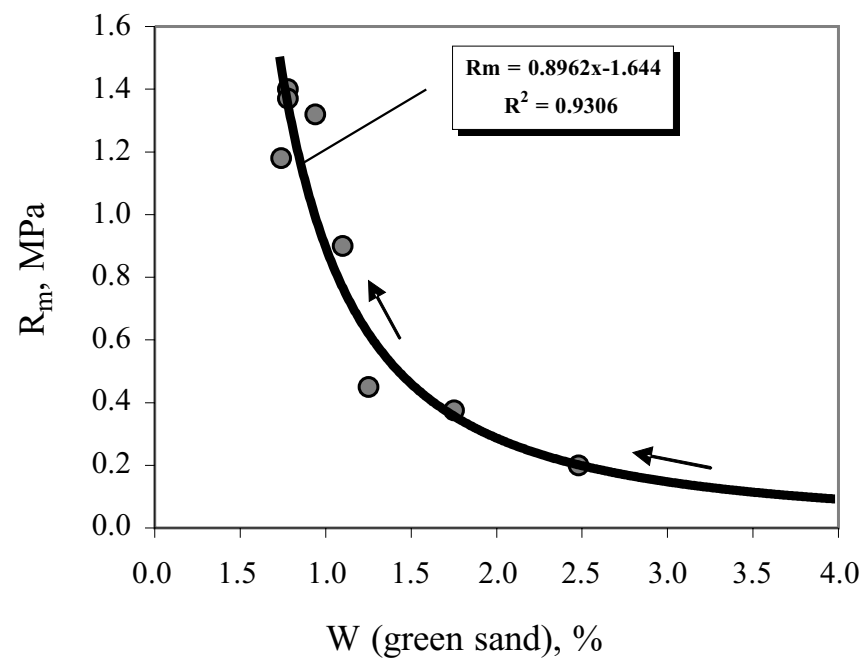

Fig. 9. Influence of the ceramic sand moisture content of the hardened mould on its tensile strength $R_{m}$, ('green'strength)

\section{CONCLUSIONS}

Investigations presented in the paper should be treated, at the current stage, as a contribution to the development of the new method of examinations the drying process of ceramic moulds used in the investment casting technology. This is the technology based on modern, environment friendly colloidal binders. Determination of time, after which it is possible to deposit the successive layer of the ceramic moulding sand, is crucial for this technology of the moulds production.

Several important conclusions can be drawn on the bases of the investigations performed by means of the weight method (recording of mass losses), by the measurements of resistance changes and by the ultrasound measurements estimating changes of reological properties of ceramic layers.

- Measurements of mass losses of the drying ceramic mould, caused by water evaporation, indicate that in the first period these losses are uniform and the drying rate is, in approximation, constant. This drying rate decreases fast in the final phase of the process, which can mean that the mechanism of the water removal has been changed. Removal of the remains ( $W=2.0-0.5 \%$ ) of unbounded water requires out-of-proportion longer time than the removal when its content is high.

- Measurements of the resistance changes during the drying period allow to divide this period into two sub-periods. In the first one, regardless of a moulding sand drying, the resistance of the ceramic layer is on a low level. In the second sub-period - when the moisture content decreases to: $W<2.0-2.5 \%$ - the resistance quite 'rapidly' increases. Large and fast increases of this resistance indicate that the drying process of the ceramic layer is near the end. 
- Ultrasound measurements describing elasticity increases of the ceramic layer also indicate the existence of two periods. In the first one - regardless of water losses the ceramic layer retains high plasticity, which results in the lack of wave velocity increases (elasticity of the investigated system). In the second period, starting from the outer surface more and more thick layer of the hardened sand is formed. This causes the wave velocity increases. After obtaining the water content: $W<2.0 \%$, further drying does not cause visible changes of the wave velocity.

- The results of the kinetics of drying in relation with the influence of the sand moisture content on its strength indicate that the moisture value $W=2.0 \%$ can be considered the limiting value in the technology of the successive layers deposition. Below this value the ceramic moulding sand is characterized: by slowing down of the drying process (further water loss), by the increasing resistance and good elastic properties - the wave velocity reaches its maximum.

The research program was performed within the statutes framework: No. 11.11.170.318. Task 4.

\section{REFERENCES}

[1] Zych J.: Synthesis of the Ultrasonic Technique Applications to Kinetics Analysis of the Selected Processes Occurring on Moulding Materials, AGH University of Science and Technology Press, Cracow 2007, Dissertations-Monographs 163, ISSN 0867-6631

[2] Zych J.: Application of the novel ultrasonic method in the on line research of the setting, and hardening process of ceramic materials, Inżynieria Materiałowa, 27 (2006) 3, pp. 680-683

[3] Zych J.: Method of examining the hardening process of core and moulding sand mixes containing a hardenable binder, Akademia Górniczo-Hutnicza: Patent G01N 29/00 (2006.01) Poland

[4] Zych J.: Moulding sands surface layer-kinetics of the changes its property, Archives of Foundry, 6 (2006) 20, pp. $77-84$

[5] Zych J.: Effecting of the weather conditions on the surface layer condition of moulds made with moulding sand whit the chemical bonding, Archives of Foundry, 6 (2006) 22, pp. 576-581 